Check for updates

Cite this: RSC Adv., 2017, 7, 36819

\title{
Millifluidic synthesis of cadmium sulfide nanoparticles and their application in bioimaging
}

\author{
Liying Hong, $\mathbb{D}$ + $\dagger^{\mathrm{ab}}$ Tai-Lok Cheung, $\dagger^{\mathrm{c}}$ Nanxi Rao, ${ }^{\mathrm{c}}$ Qingling Ouyang, ${ }^{\text {ad }}$ Yue Wang, ${ }^{\mathrm{e}}$ \\ Shuwen Zeng, ${ }^{\text {ad }}$ Chengbin Yang, ${ }^{\text {ab }}$ Dang Cuong, ${ }^{a}$ Peter Han Joo Chong, ${ }^{*}{ }^{*}$ \\ Liwei Liu, (DD *e Wing-Cheung Law ${ }^{* c}$ and Ken-Tye Yong ${ }^{\star a b}$
}

In this paper, a miniature fluidic synthesis platform utilizing millimeter dimension channels for the synthesis of cadmium sulfide (CdS) quantum dots and nanocrystals is demonstrated. Traditional nanoparticle synthesis techniques involve macroscopic flasks where reaction conditions may vary at different positions inside the vessel. Therefore challenges in terms of batch reproducibility for large scale production are of great concern. Here, we show that it is possible to replicate reaction conditions so as to produce nanoparticles with similar optical characteristics across different batches using the same reaction parameters. Particle size control was established by varying the flow rate of the precursors, yielding gradually increasing nanocrystal sizes from $2.4 \mathrm{~nm}$ to $3.7 \mathrm{~nm}$ with increasing residence time. The as-synthesized CdS nanoparticles exhibited tunable photoluminescence by adjusting the molar ratio of the cadmium and sulfur precursors, giving rise to greenish-blue and orange-red emissions under ultraviolet light illumination. The particles were then studied and characterized using transmission electron microscopy (TEM), ultraviolet-visible (UV-Vis) absorbance, photoluminescence, X-ray diffraction (XRD) and selected area electron diffraction (SAED) techniques. Lastly, bioimaging of RAW264.7 mice macrophage cells using ligand exchanged CdS nanoparticles is presented.

Received 12th May 2017

Accepted 15th July 2017

DOI: 10.1039/c7ra05401g

rsc.li/rsc-advances of light on CdS nanoparticles with ruthenium(rv) oxide catalyst. Following which many preparation methods such as hot-injection, ${ }^{5,6}$ solvothermal,, 78 non-injection ${ }^{9,10}$ and microwave-assisted synthesis $^{11,12}$ emerged over time. However, some of these techniques involve the use of elevated temperatures, pressures, highly explosive organometallic compounds, and long reaction durations, resulting in differences in terms of particle size, morphology and crystallinity of the CdS nanoparticles. Therefore, we believe that miniature reaction vessels can help to improve the quality, reproducibility and ease of production of luminescent CdS nanocrystals (NCs).

CdS semiconductor nanoparticles are of interest in various applications such as optoelectronics particularly in photovoltaics and photoactivation, ${ }^{13-16}$ bioimaging ${ }^{17}$ and biosensing. ${ }^{18}$ At the nanoscale where quantum confinement effects come into play, these nanoparticles can exhibit very different optical and chemical properties from its bulk material. CdS nanocrystals exhibit rich morphologies, for example spheres, nanorods, nanocubes, tripods, tetrapods, candy corn, and arrow-shaped..$^{5,19}$ Therefore, understanding and controlling of the shapes, sizes and surface characteristics of CdS nanoparticles are crucial for developing devices with unique properties like quantum dot-sensitized solar cells with higher stability than thin-film based solar cells made using the same materials. ${ }^{20}$

Miniature reaction chambers provide the advantages of a well-controlled environment by means of reproducible mixing 
conditions enabled by manipulation of laminar fluid flow in engineered channel designs or active micromixers, ${ }^{28,29}$ excellent heat and mass transfer due to increased surface area to volume ratio $^{30}$ provided by the miniature channel dimensions. In addition, on-chip synthesis provides a spatial-to-temporal mapping of the reaction dynamics. ${ }^{31}$ Traditional macroscale QD fabrication methods such as organometallic synthesis involve the usage of highly toxic precursors and generation of considerable chemical waste like solvents, surfactants, unreacted precursors and unwanted reaction by-products. On the other hand, microfluidic and millifluidic synthesis methods are greener approaches as the quantity of required chemicals are much reduced and the homogenous reaction conditions result in lesser waste products due to more efficient reaction kinetics. ${ }^{32}$

Due to the potential advantages of miniature systems, many groups have also explored CdS nanoparticle synthesis using miniature reaction vessels such as capillary tubes, microreactors, microfluidic chips, and even microorganisms like bacteria (Table 2). Although there is great promise in these minuscule technologies, they are still under development and have different limitations which need to be tackled. For example, microscale systems involving capillary tubes offer simplicity in terms of implementation and adaptation to macroscale injection methods, but are constrained by limited geometric designs and relatively larger footprints. ${ }^{33}$ Biosynthesis methods, on the other hand, despite enabling the fabrication of biocompatible nanoparticles using green chemistry at room temperatures, suffers from low throughput, long synthesis durations, ${ }^{27}$ and requires further investigation of the nanoparticle purification processes as biomolecules might still be attached to the nanoparticle surfaces. Miniature fluidic channels or lab-on-chip (LoC) devices, in addition to the advantages offered by capillary tubes, provide additional flexibility for various designs to generate reproducible mixing within the channel. However, most of the existing on-chip synthesis of CdS nanoparticles are based on aqueous solvents with reaction temperatures around $25-30{ }^{\circ} \mathrm{C}$, yielding poor crystalline nanoparticles.

In this work, controlling the size and morphology of CdS nanocrystals (NCs) in a millifluidic chip was demonstrated. By varying the injection flow rate and precursor ratio, spheres, nanorods and multipods with sizes ranging from $2.6 \mathrm{~nm}$ to $6.7 \mathrm{~nm}$ were obtained. The NCs synthesized in less than $3.5 \mathrm{~min}$ exhibited good crystallinity as substantiated by the high resolution transmission electron microscope (HRTEM) images as well as the powder X-ray diffraction (XRD) patterns. Size variations along with changes in the optical properties of these assynthesized CdS NCs were also achieved by varying the residence time, and molar ratios of the precursors. Here, we employed oleylamine (OAm) as both the surfactant and coordinating solvent for the reaction. Oleylamine has been successfully proven to control shape and size of nanocrystals in traditional benchtop methods as demonstrated by Yong et al. ${ }^{5}$ and Joo et al., ${ }^{19}$ where elemental sulfur was added to metaloleylamine complexes to form semiconducting metal sulfide nanoparticles. Therefore, we believe that there is great potential in extending their use in millifluidic synthesis methods. We first discuss the effects of chip residence time on the NCs formed, followed by examining the effect of varying the molar ratios of the $\mathrm{Cd}$ and $\mathrm{S}$ precursors. Lastly, the millifluidic chip synthesized CdS NCs were employed in a proof of concept demonstration for in vitro bioimaging using RAW264.7 mice macrophage cells.

\section{Experimental}

\section{Materials}

Cadmium chloride and sulfur (Sigma Aldrich), oleylamine ( $>50.0 \%$, Tokyo Chemical Industry Co., Ltd.). Toluene ( $>99.5 \%$, Chemicals Testing and Laboratory, Singapore), ethanol (95\%, Aik Moh Pte Ltd, Singapore) were used in the synthesis of cadmium sulfide nanoparticles. The 184 Sylgard silicone elastomer kit from Dow Corning was used to fabricate the polydimethylsiloxane (PDMS) millifluidic chip. RAW264.7 mice macrophage cell line (ATCC, Manassas, VA, USA), Dulbecco's Modified Eagle's Medium ([DMEM], Hyclone), fetal bovine serum ([FBS], Hyclone), penicillin-streptomycin (Gibco, Life Technologies, SG, Singapore) were used for the cell culture. All the above chemicals were used as purchased without further purification. Deionized (DI) water mentioned in the experiments was purified by a Milli-Q water purification system.

\section{Fabrication of millifluidic chip}

The millifluidic channels were designed using the free software SketchUp and the mould was printed in acrylonitrile butadiene styrene (ABS) material using the UP Plus 2 3D printer (PP3DP). The Sylgard 184 Elastomer Kit was used for the polydimethylsiloxane (PDMS) mixture based on a $10: 1$ ratio of the base resin to the curing agent. The base and curing agent were then mixed homogeneously before degassing the mixture in a dessicator to remove the bubbles. Following that, the PDMS mixture was poured into the mould, degassed and heat cured on a hotplate at $70{ }^{\circ} \mathrm{C}$ for at least 4 hours. Subsequently, the PDMS was carefully removed from the mould and bonded to a glass slide. Holes were then made at the inlets and outlet of the chip and polytetrafluoroethylene (PTFE) tubes used to deliver the precursors and nanocrystal product were inserted and sealed to the chip.

\section{Synthesis of CdS nanocrystals in millifluidic chip}

The synthesis protocol used is similar to ref. 5 and 19. Briefly, $6 \mathrm{mmol}$ of sulfur powder was dissolved in $10 \mathrm{ml}$ of oleylamine and stirred at room temperature in a flask until all the powder has completely dissolved. In another three neck flask, $1 \mathrm{mmol}$ of cadmium chloride $\left(\mathrm{CdCl}_{2}\right)$ was dissolved in $10 \mathrm{ml}$ of oleylamine, heated and stirred at $170{ }^{\circ} \mathrm{C}$ under nitrogen $\left(\mathrm{N}_{2}\right)$ flow for 20 minutes, before the temperature was raised further to $200{ }^{\circ} \mathrm{C}$ for another 10 minutes. Both the sulfur-oleylamine (S-OAm) and the cadmium-oleylamine (Cd-OAm) mixtures were then withdrawn using separate syringes and injected into the PDMS millifluidic chip. 
The precursors were delivered into the millifluidic chip via the syringe pump (KD Scientific), where the injection flow rates of the precursors were set at $5000 \mu \mathrm{lmin}^{-1}, 3000 \mu \mathrm{l} \mathrm{min}^{-1}, 1500$ $\mu \mathrm{lmin} \mathrm{m}^{-1}, 750 \mu \mathrm{l} \mathrm{min}{ }^{-1}$ and $375 \mu \mathrm{min}^{-1}$ and the products of the reaction was collected at the outlet of the chip. In addition, different molar ratios of the precursors were also used. The molar ratios of cadmium is to sulfur $(\mathrm{Cd} \mathrm{:S})$ were varied from $1: 3\left(1 \mathrm{mmol}\right.$ of $\mathrm{CdCl}_{2}$ and $3 \mathrm{mmol}$ of $\left.\mathrm{S}\right), 1: 6$, and $1: 12$ ( $1 \mathrm{mmol}$ of $\mathrm{CdCl}_{2}$ and $12 \mathrm{mmol}$ of $\mathrm{S}$ ). Toluene was added external to the chip in the collecting vial to quench the reaction and stop further growth of the nanocrystals. To purify the CdS NCs, 2 parts of ethanol were added to 1 part of the output solution and mixed before centrifuging at $8000 \mathrm{rpm}$ for 3 minutes. The supernatant was decanted and the pellet was redispersed in toluene for subsequent storage of the NCs.

\section{Characterization of CdS nanocrystals}

The ultraviolet-visible (UV-Vis) absorption spectrum of the CdS nanocrystals was measured using a Shimadzu UV-2450 spectrophotometer, while the photoluminescence emission spectrum was collected using a HORIBA Jobin Yvon Fluorolog-3 Fluorometer. Transmission electron microscope (TEM) images were obtained using a JEOL JEM-2011 TEM. The Rigaku SmartLab powder X-ray diffractometer was used to carry out the powdered X-ray diffraction (XRD) measurements to determine the crystallographic structures present.

\section{Ligand exchange of CdS nanocrystals with mercaptosuccinic acid}

For $5 \mathrm{mg}$ of CdS NCs with oleylamine ligands, $150 \mathrm{mg}$ of mercaptosuccinic acid (MSA) should be used. The MSA was dissolved in $3 \mathrm{ml}$ of chloroform with stirring, then the assynthesized CdS with oleylamine ligands from the previous section was added. Subsequently, ammonium hydroxide $\left(\mathrm{NH}_{3} \cdot \mathrm{H}_{2} \mathrm{O}\right)$ and deionized water (DI $\left.\mathrm{H}_{2} \mathrm{O}\right)$ were added to the mixture in a volumetric ratio of $3: 7$ and stirred vigorously overnight. When the ligand exchange process is completed, the mixture should exhibit two distinct layers with the yellow CdS NCs as the top layer and a clear bottom layer. Extract the top layer carefully and flocculate the NCs using ethanol, decant the supernatant and dissolve the pellet in deionized water for further use.

\section{Cell culture and bioimaging of macrophage cells using millifluidic CdS nanocrystals}

RAW264.7 mice macrophage cells (American Type Culture Collection) were cultured with Dulbecco's modified eagle's medium (DMEM, Hyclone), supplemented with 10\% FBS, penicillin $\left(100 \mu \mathrm{g} \mathrm{ml}^{-1}\right.$, Gibco $)$ and streptomycin $\left(100 \mu \mathrm{g} \mathrm{ml}^{-1}\right.$, Gibco $)$ in an incubator at $37{ }^{\circ} \mathrm{C}$ and $5 \% \mathrm{CO}_{2}$. The cells were seeded at a density of $1 \times 10^{5}$ cells per well with DMEM culture medium. $3.8 \mathrm{mg} \mathrm{ml}^{-1}$ of CdS-MSA were added to the macrophage cells and incubated for 4 hours. Then, the treated cells were rinsed thrice with PBS buffer $(\mathrm{pH}=7.2)$ and fixed with $4 \%$ formaldehyde. In vitro microscope images were obtained using a fluorescence microscope (Eclipse Ti-U Inverted Microscope System, Nikon) and a filter cube with $350 \mathrm{~nm}$ excitation wavelength and a red emission filter with a centre wavelength of $630 \mathrm{~nm}$.

\section{Results \& discussion}

The dimensions of the channel on the miniature chip is $1.5 \mathrm{~mm}$ (width) by $1.5 \mathrm{~mm}$ (height), spanning a total length of approximately $545 \mathrm{~mm}$ and capable of carrying a total fluid capacity of $1227 \mathrm{~mm}^{3}$ or $1227 \mu \mathrm{l}$. Due to the millimeter scaled channel, we term the chip as a millifluidic chip. There are five sections constituting this chip. Two inlets for the precursor solutions and one outlet are depicted in Fig. 1b. The sulfur and cadmium precursors are injected into the chip via separate inlets before the two streams meet at the Y-shaped region. Next, the streams encounter a winding section, which folds the fluids and promotes mixing ${ }^{34}$ as illustrated by the flow velocity profile in Fig. 1d. After mixing, the solution then flows into the straight regions where nucleation and growth take place to form the CdS NCs indicated by the increase in CdS concentration simulated in Fig. 1c. Finally, the reaction is quenched as it flows out of the chip outlet into the cold toluene solution.

The base of the millifluidic chip is bonded to a glass slide to allow uniform heating of the entire chip and reduce temperature fluctuations of particles at different regions of the chip. Our continuous flow fluidic chip boasts a simple passive mixing environment providing stable conditions for nanoparticle incubation. Unlike traditional flask synthesis techniques for CdS nanoparticles, where it is necessary to flush the reaction vessel with nitrogen gas ${ }^{5,19}$ to avoid oxidation of the nanoparticles, this millifluidic synthesis platform enables mixing of the precursors within the chip without the need to purge the channels with inert gases. ${ }^{35}$ The inert environment is only required in the precursor preparation stage, prior to mixing of both precursors.

Examples of CdS NCs with different morphologies and sizes synthesized using our millifluidic chip are shown in Fig. 2. By varying parameters such as the injection flow rate, dominantly spherical particles could be obtained with sizes ranging from 2.4-5.8 $\mathrm{nm}$. In addition, morphologies such as tripods and

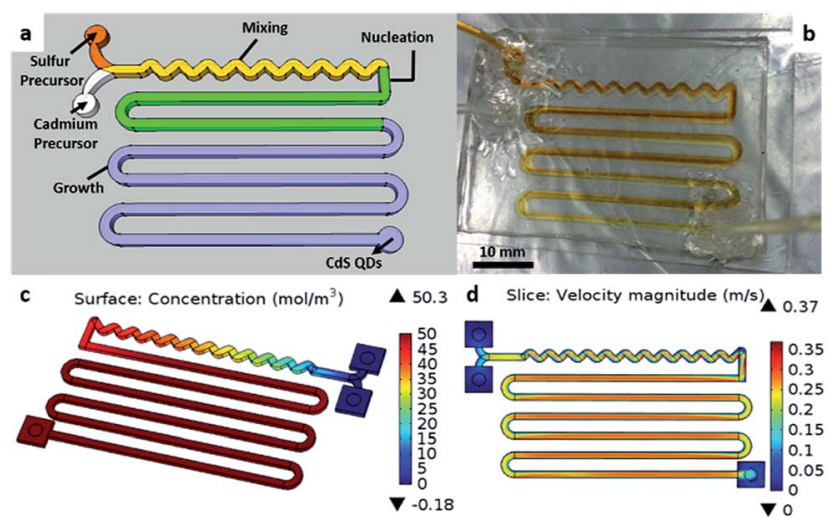

Fig. 1 (a) Annotated schematic and (b) actual millifluidic chip for the synthesis of cadmium sulfide nanocrystals (CdS NCs). Simulations reflecting CdS NC (c) concentration and (d) fluid velocity flow inside the chip. 


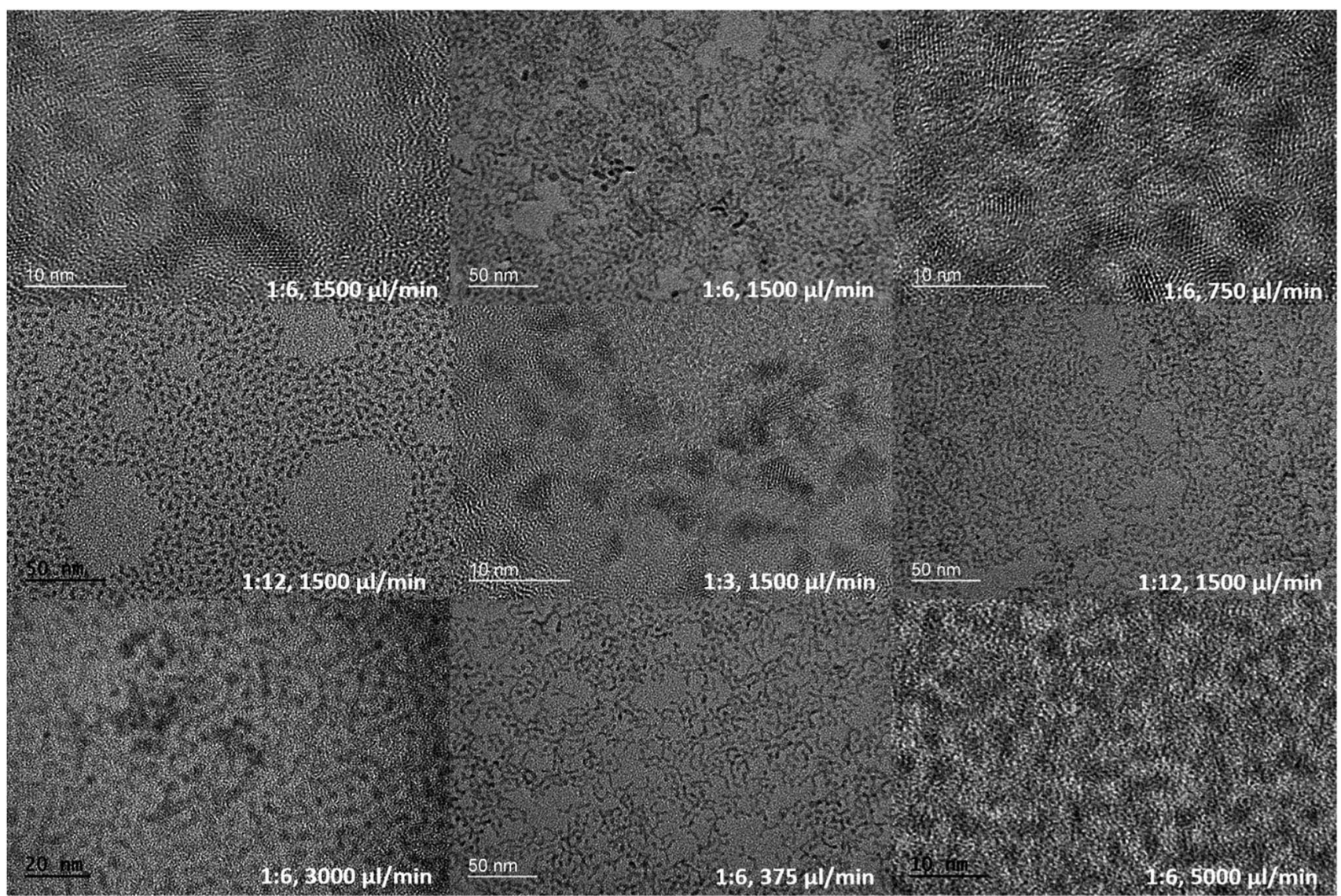

Fig. 2 TEM images of CdS NCs produced under different flow rates and Cd:S precursor molar ratios.

bipods were also obtained by changing the flow rate and the $\mathrm{Cd}: \mathrm{S}$ ratios.

\section{Effect of injection flow rate/residence time}

Different injection flow rates were studied to examine their effects on the formation of CdS NCs. The injection flow rate determines the residence time $\left(\tau_{R}\right)$ of the fluid in the chip. Because the reaction time in bulk macroscale synthesis methods cannot be directly converted into the residence time in the chip, ${ }^{36}$ studies involving short reactions times can be made possible by utilizing miniature reactors. Compared to conventional bench top synthesis using the same reactants, ${ }^{5,19}$ our
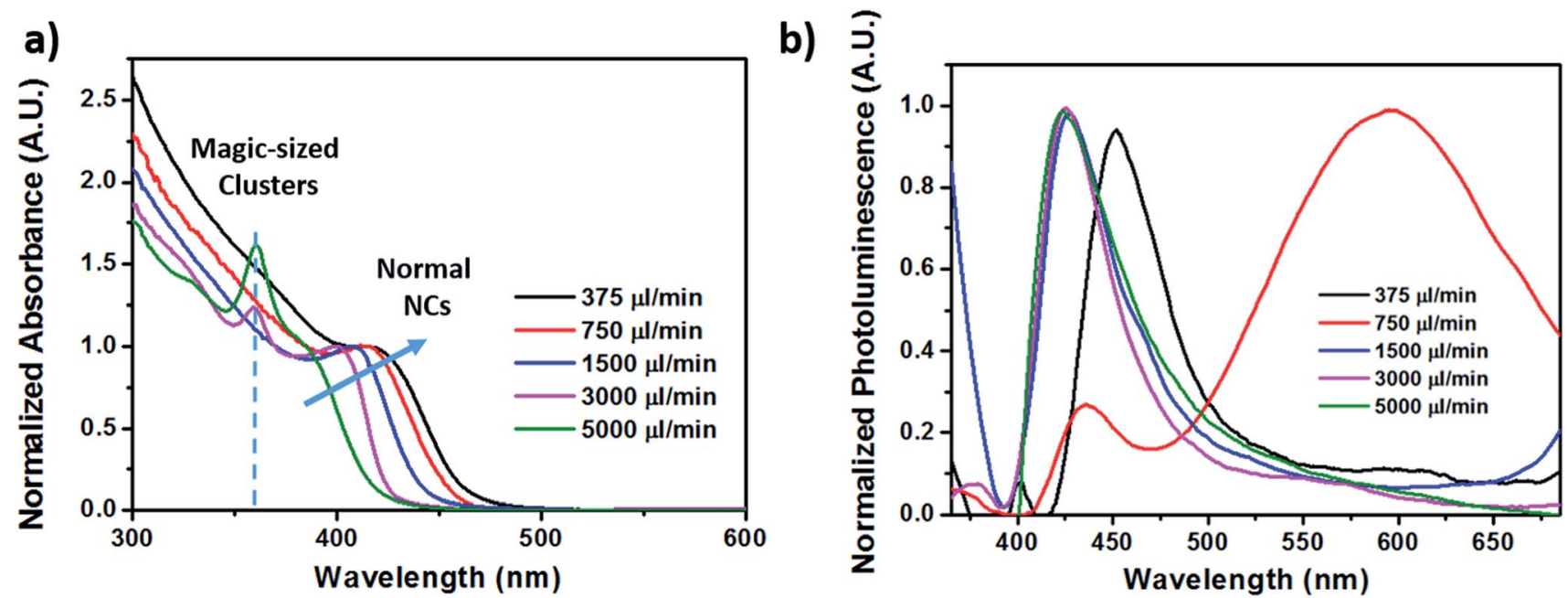

Fig. 3 (a) Absorption curves and (b) photoluminescence spectra of CdS NCs formed at different flow rates with Cd: S molar ratio fixed at 1 : 6. 


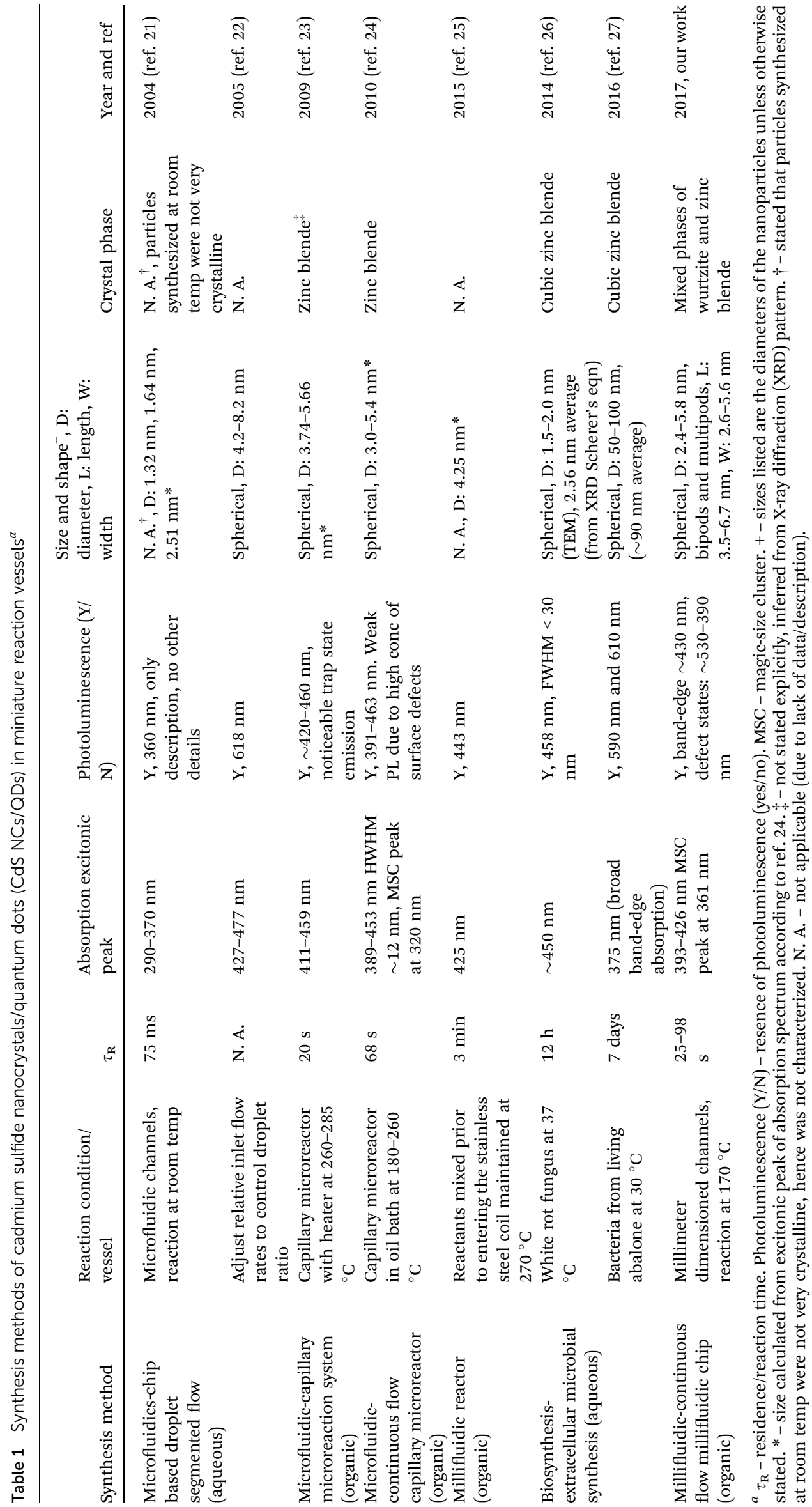




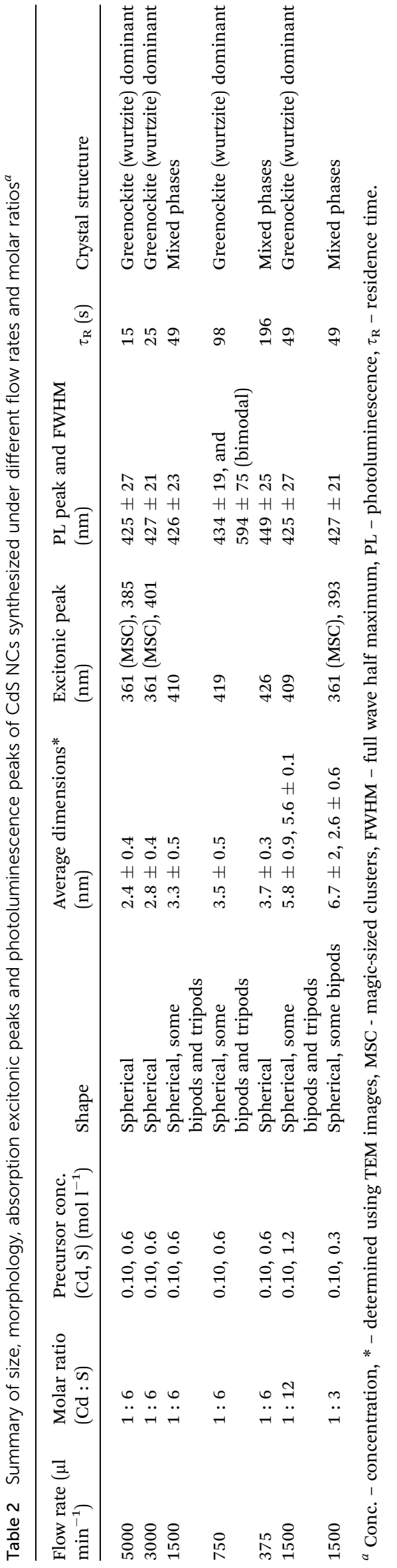

homemade millifluidic chip was set with flow rates of $5000 \mu \mathrm{l}$ $\min ^{-1}$ and $3000 \mu \mathrm{min}^{-1}$, corresponding to a residence time of about $15 \mathrm{~s}$ and $25 \mathrm{~s}$ respectively, produced quantum-confined NCs or quantum dots with an average size of $2.4 \pm 0.4 \mathrm{~nm}$ and $2.8 \pm 0.4 \mathrm{~nm}$ (Fig. 6a-d), bordering the exciton Bohr radius of CdS. Upon decreasing the flow rate to $1500 \mu \mathrm{l} \mathrm{min}{ }^{-1}, 750 \mu \mathrm{l}$ $\min ^{-1}$, and $375 \mu \mathrm{min}^{-1}$, larger nanocrystals were obtained with average diameters of $3.3 \pm 0.5 \mathrm{~nm}$ (Fig. 6e and f), $3.5 \pm$ $0.5 \mathrm{~nm}$ (Fig. $6 \mathrm{~g}$ and h), and $3.7 \pm 0.3 \mathrm{~nm}$ (Fig. 6i and j) respectively. Based on the TEM images in Fig. 2, the shape of the CdS NCs were varied (i.e. spherical, bipod and tripod) by altering the injection flow rate.

The ultraviolet-visible (UV-Vis) absorption curves in Fig. 3a show the first excitonic peaks for the CdS NCs synthesized at different flow rates. Based on empirical studies by Yu et al., ${ }^{37}$ the size of the NCs can be estimated from the position of the excitonic peaks. The absorption spectra in Fig. 3a further corroborates the particle sizes determined from the TEM images with peak values at $385 \mathrm{~nm}, 401 \mathrm{~nm}, 410 \mathrm{~nm}, 419 \mathrm{~nm}$, and $426 \mathrm{~nm}$ corresponding to flow rates of $5000 \mu \mathrm{min}^{-1}, 3000 \mu \mathrm{l} \mathrm{min}{ }^{-1}$, $1500 \mu \mathrm{l} \mathrm{min}{ }^{-1}, 750 \mu \mathrm{min}^{-1}$, and $375 \mu \mathrm{min}^{-1}$ respectively. The size of these NCs determined from our TEM images (Table 1) agree largely with the empirical estimation derived by Yu et al. ${ }^{37}$ varying on an average of $0.5 \mathrm{~nm}$. The longer residence time due to slower injection flow rates enabled the particles to increase in size as a result of the longer growth period.

Besides that, a consistent additional peak was observed at $361 \mathrm{~nm}$ for the absorption spectra of CdS NCs synthesized at fast flow rates of $5000 \mu \mathrm{l} \mathrm{min}{ }^{-1}$ and $3000 \mu \mathrm{l} \mathrm{min}{ }^{-1}$, exhibiting linewidths as narrow as $14 \mathrm{~nm}$. The presence of this discrete peak indicates the formation of a magic-size cluster (MSC), which degrades in intensity over time before spontaneously redshifting to another distinct wavelength instead of gradually redshifting over time. ${ }^{38}$ This is in stark contrast to the red-shifted peak at the longer wavelength region, which progressively shifted from $385 \mathrm{~nm}$ to $426 \mathrm{~nm}$ as the residence time increased from $15 \mathrm{~s}$ to $196 \mathrm{~s}$, while the MSC peak remained constant at $361 \mathrm{~nm}$ as indicated in Fig. 3a. In addition, we demonstrated the high reproducibility for synthesizing highly monodispersed CdS NCs under the flow rate of $5000 \mu \mathrm{min}^{-1}$ by showing that the same MSC peak at $361.7 \pm 0.5 \mathrm{~nm}$ (Fig. 4a) could be replicated. Fig. 4 also shows other synthesis parameters which exhibited almost identical absorption spectra, providing compelling evidence for the reliability and stability of the millifluidic chip platform as a miniature reaction vessel.

These as-synthesized CdS NCs emitted visible light when irradiated with ultraviolet (UV) light with an excitation wavelength of $350 \mathrm{~nm}$. From Fig. 3b, NCs produced using the slower flow rate of $750 \mu \mathrm{min}^{-1}$ exhibited a bimodal emission with a longer wavelength peak at around $594 \mathrm{~nm}$, along with a shorter wavelength peak corresponding to the band edge emission at $434 \mathrm{~nm}$. The intensity of the intensity of the longer wavelength peak was much higher than that of the shorter wavelength peak. Also, the $594 \mathrm{~nm}$ peak had a broader linewidth compared to the $434 \mathrm{~nm}$ peak. Meanwhile, particles fabricated using the high flow rates of $1500 \mu \mathrm{min}^{-1}$ and above 
a)

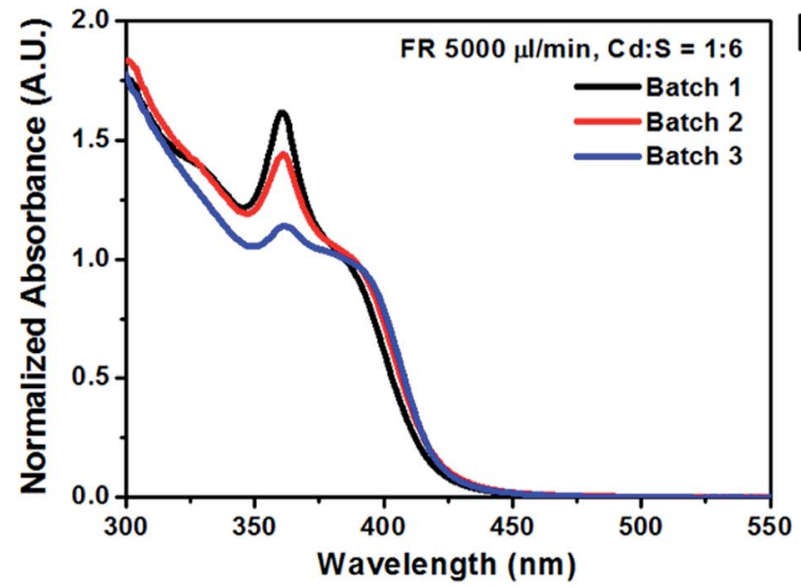

c)

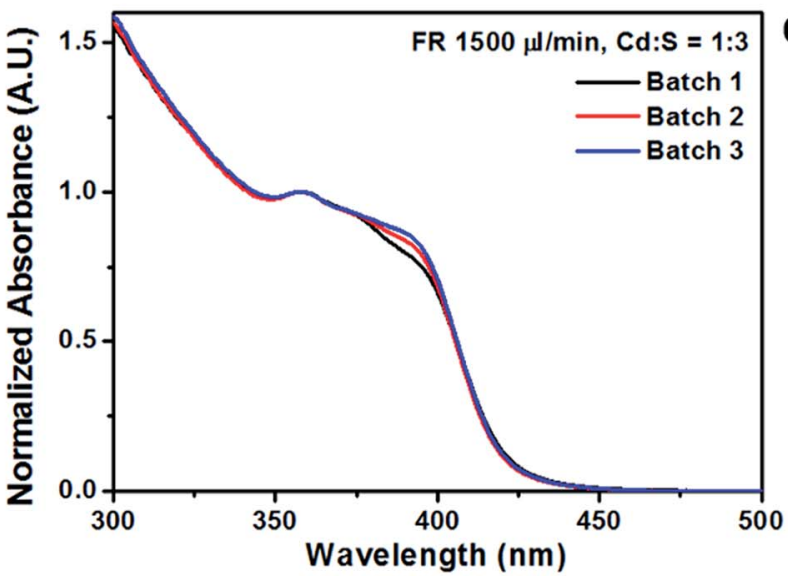

b)

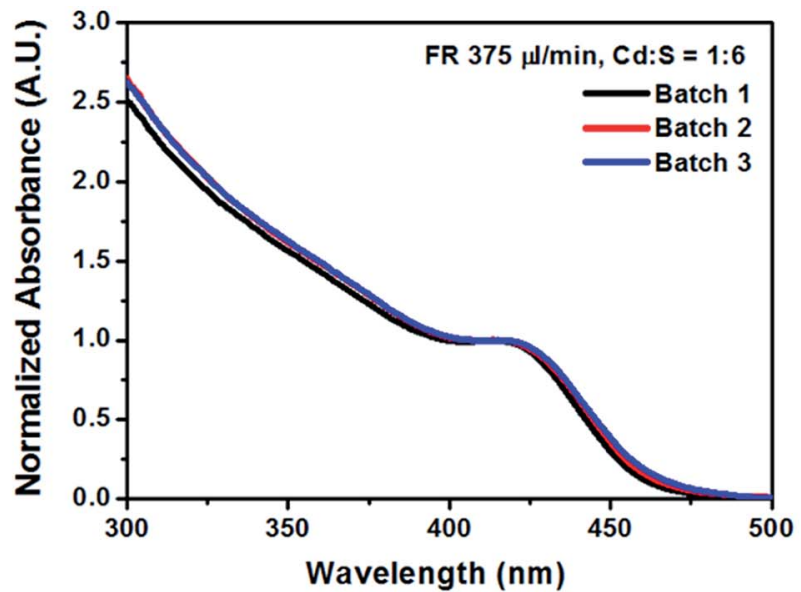

d)

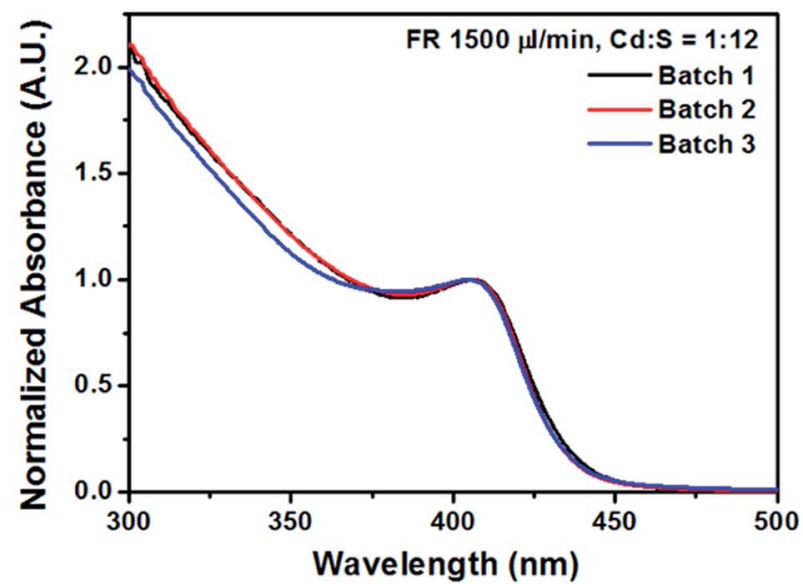

Fig. 4 Absorption curves of selected batches of CdS NCs showing the high reproducibility of nanoparticle fabrication using these millifluidic chip reactors.

had a dominant peak at the band edge $(\sim 427 \mathrm{~nm})$ with a trailing edge spreading over to the longer wavelength region.

For this set of CdS NCs (i.e. with varying flow rates), the molar ratio of $\mathrm{Cd}$ and $\mathrm{S}$ precursors were fixed at $1: 6$, with $\mathrm{S}$ being in excess. The red-shifted broadband photoluminescence emission depicted in Fig. 5 is attributed to the sulfur defect states. ${ }^{39}$ Incomplete passivation of the CdS nanocrystals with oleylamine ligands resulted in dangling bonds at the nanocrystal surface and thus caused surface trap states to exist within the nanocrystal band gap. These surface states then affect the relaxation process of the carriers, resulting in nonradiative pathways which reduce the energy of the emitted photon upon carrier recombination, thereby causing the red shift and broadband spectral features of the photoluminescence. This broadband emission has been commonly observed for such small sized CdS quantum dots and nanocrystals. . $^{722,23,39,40}$

\section{Effect of different Cd : S molar ratios}

In addition to the initial $\mathrm{Cd}: \mathrm{S}$ precursor molar ratio of $1: 6$, the influence of the relative ratios of cadmium and sulfur on the size and morphology of the NCs was also studied. Two additional Cd : S precursor molar ratios were employed, making the range of molar ratios investigated $-1: 3,1: 6$ and $1: 12$. In all three cases, the ratio of 1 corresponded to $0.1 \mathrm{M}$ of the cadmium precursor. Fig. 7 depicts the TEM images of the NCs formed at a fixed flow rate of $1500 \mu \mathrm{l} \mathrm{min}{ }^{-1}$ but with different molar ratios of $1: 3$ (Fig. 7a-c), $1: 6$ (Fig. 7d-f), $1: 12$ (Fig. 7g-i) together with their size distributions. Attempts were made to put the ratio of the Cd precursor in excess, however they were unsuccessful as

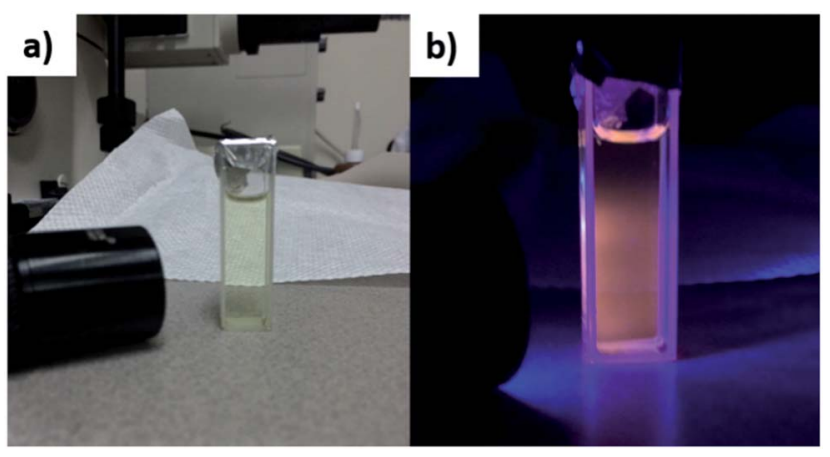

Fig. 5 Millifluidic (mF) CdS NCs under (a) ambient light or (b) ultraviolet (UV) irradiation, with the reddish glow of $\mathrm{mF} \mathrm{CdS}$ defect state emission in the dark. 

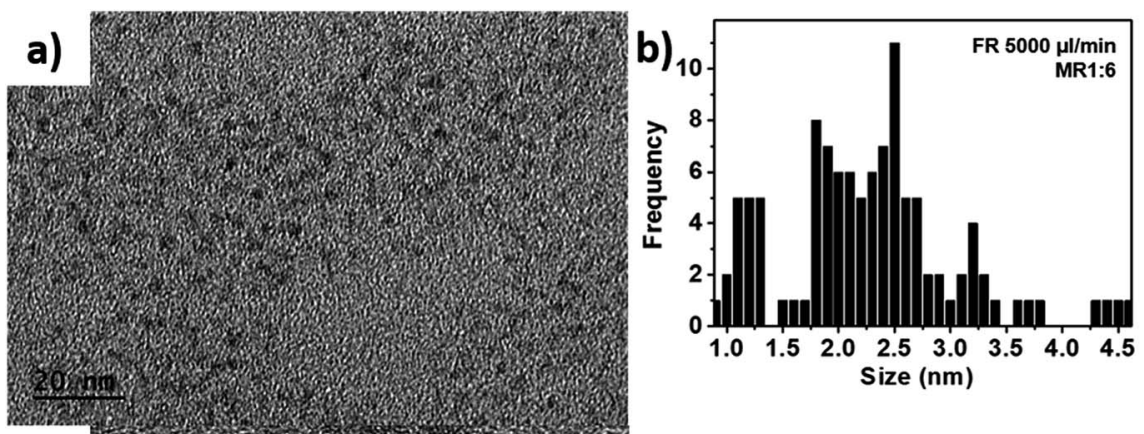

\section{c)}

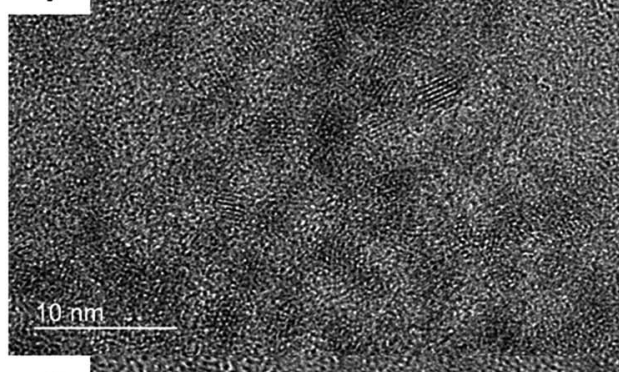

e)

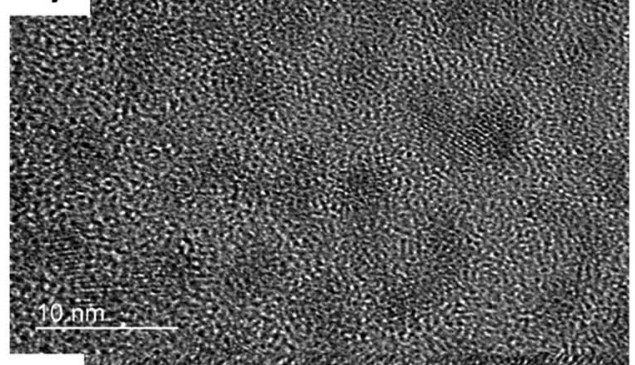

g)
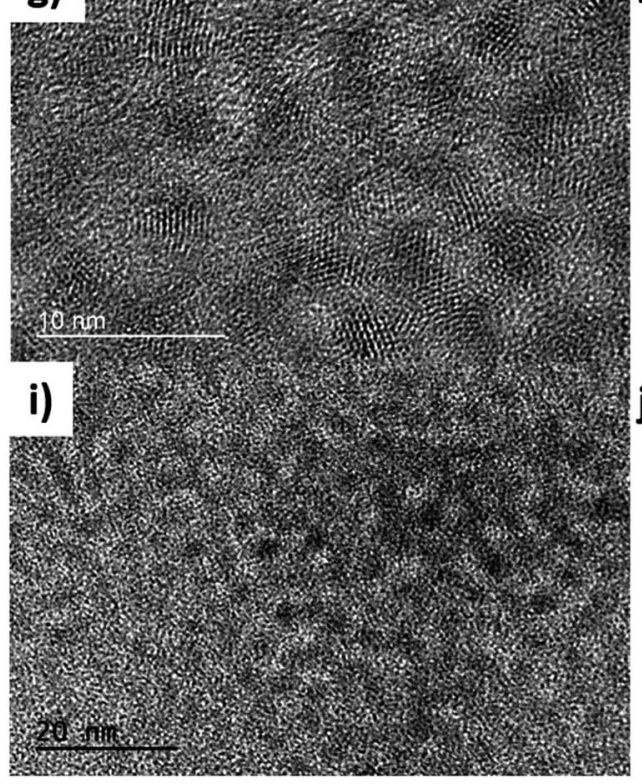

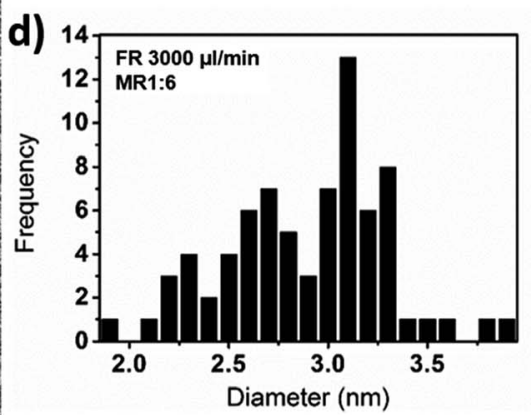

f)
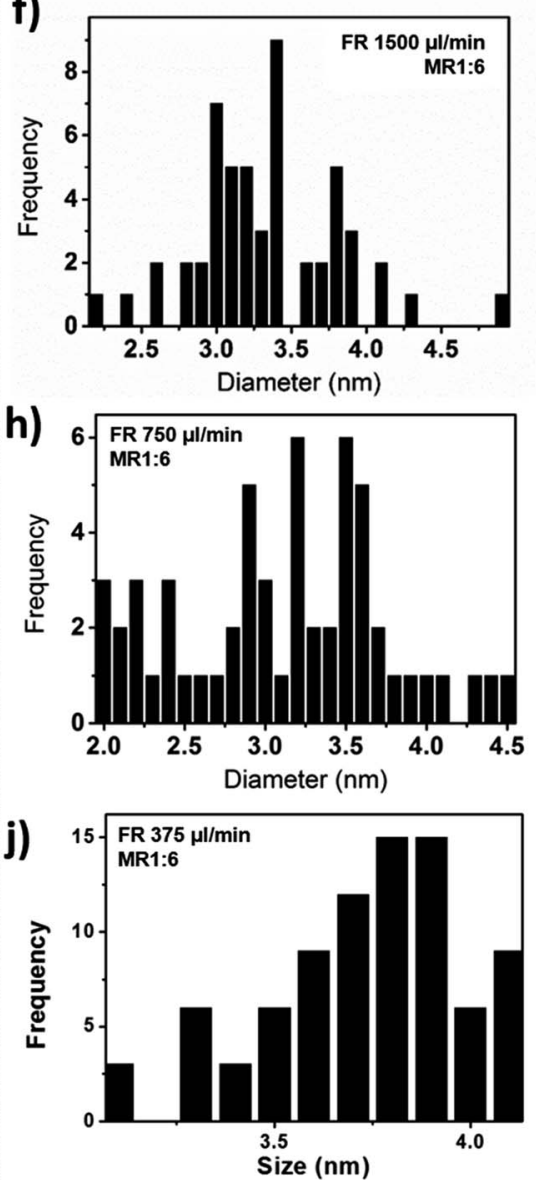

Fig. 6 TEM images and size distributions of CdS nanocrystals synthesized in millifluidic chips at different flow rates of $5000 \mu \mathrm{l} \mathrm{min}{ }^{-1}$ (a and b),

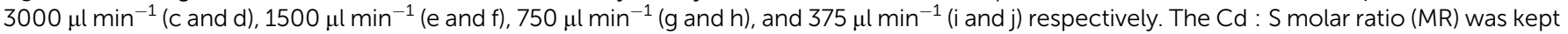
constant at $1: 6$. 
$\mathrm{Cd}: S=1: 3,1500 \mu \mathrm{l} / \mathrm{min}$

a)

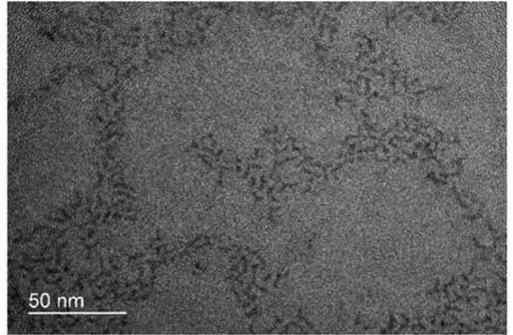

$C d: S=1: 6,1500 \mu \mathrm{l} / \mathrm{min}$

d)

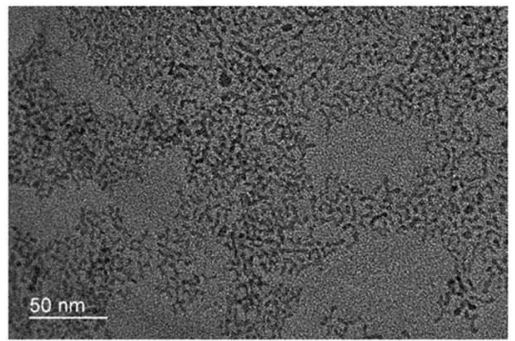

$C d: S=1: 12,1500 \mu \mathrm{l} / \mathrm{min}$

g)

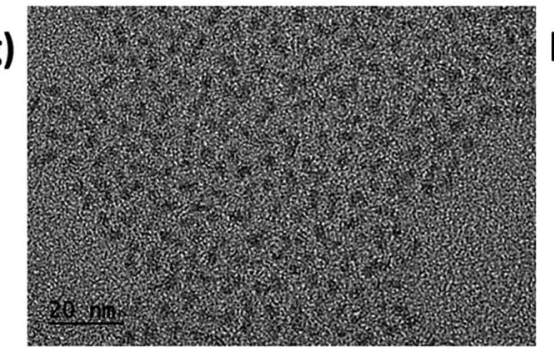

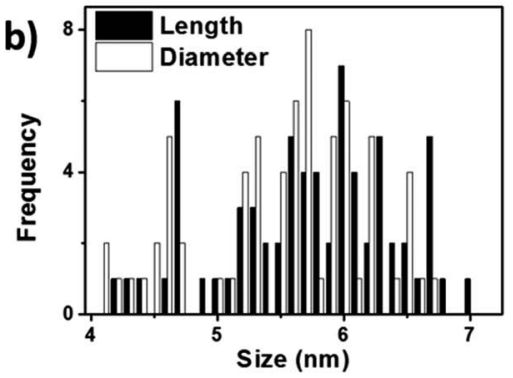
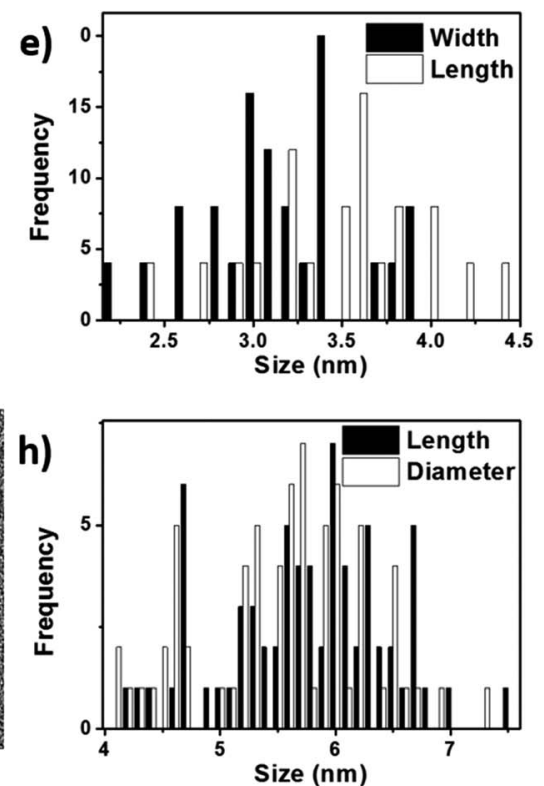
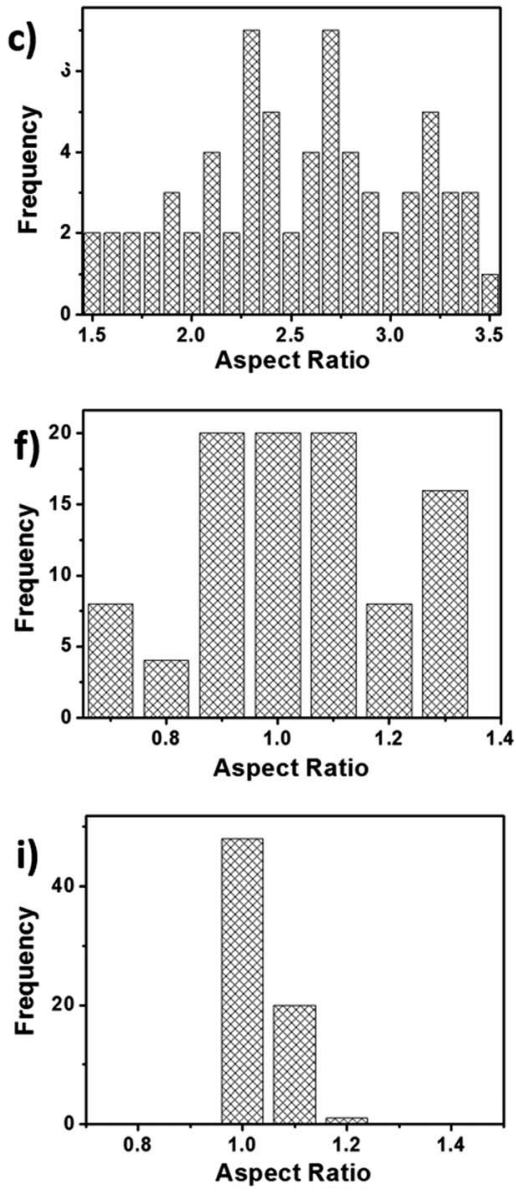

Fig. 7 TEM images of CdS nanocrystals fabricated using millifluidic chips at three different molar ratios of (a) Cd:S=1:3 (0.10 M of Cd and 0.3 $\mathrm{M}$ of S), (d) $\mathrm{Cd}: \mathrm{S}=1: 6(0.10 \mathrm{M}$ of $\mathrm{Cd}$ and $0.6 \mathrm{M}$ of S), and (g) Cd : S=1:12 (0.05 M of Cd and $0.3 \mathrm{M}$ of S). Their corresponding size distributions $(b, e, h)$ and aspect ratios $(c, f, i)$ are also presented. The flow rate was kept constant at $1500 \mu \mathrm{lmin}^{-1}$.

the precursors precipitated very quickly, causing blockages in the tubes and channels. Therefore, the change in molar ratio was only confined to increasing the amount of sulfur used in the reaction.

Based on the TEM images in Fig. 7, it was observed that the molar ratio of $\mathrm{Cd}: \mathrm{S}(1: 3)$ gave rise to substantial amounts of tripod structures having average arm lengths of $6.7 \pm 2 \mathrm{~nm}$ and diameters of $2.6 \pm 0.6 \mathrm{~nm}$ and aspect ratios of $2.58 \pm 0.6$. The molar ratio of $1: 6$ yielded a mixture of spherical and multipod structures. For the extreme case of $\mathrm{Cd}: \mathrm{S}=1: 12$, large particles with bipod shapes were observed. The absorption and photoluminescence spectra of the CdS NCs synthesized using the Cd : S molar ratio of $1: 3$ showed that these NCs exhibited different optical characteristics from that of the $1: 6$ and $1: 12$ cases. Along with larger particle sizes when $\mathrm{Cd}: \mathrm{S}=1: 3$, a bimodal fluorescence peak consisting of the narrow band edge emission at $434 \mathrm{~nm}$ and a broadband defect emission at the longer wavelength of $594 \mathrm{~nm}$ (Fig. 9) was also obtained.

Fig. $8 \mathrm{~b}$ shows the greenish-blue emissions of the millifluidic prepared CdS NCs under UV light illumination, which is different from the orange-red emission (due to surface defect states) in Fig. 5. This greenish-blue photoluminescence (PL) is due to the band edge emission of the CdS NCs. Comparing the photoluminescence spectrum in Fig. $3 \mathrm{~b}$ and $9 \mathrm{~b}$, both show the presence of bimodal fluorescence peaks. However, when the molar ratio (MR) of $\mathrm{Cd}$ and $\mathrm{S}$ was varied such that $\mathrm{Cd}: \mathrm{S}=1: 3$ (Fig. 9b), the relative PL intensity of the narrow linewidth band edge emission was almost comparable to that of the broadband surface defects emission, while for the case of sulfur being in larger excess with MR of $1: 6(\mathrm{Cd}: \mathrm{S})$, the band edge emission was much weaker compared to the emission due to surface defect states (Fig. 3b). In a study by Wei et al. ${ }^{39}$ they showed that when the CdS QDs were terminated with sulfur, the band edge photoluminescence was quenched, giving rise to little or no emission, while cadmium terminated CdS QDs exhibited higher photoluminescence and appeared brighter. Therefore, due to the excess sulfur precursors employed for MR $1: 6$, it was likely that the band edge emission was significantly quenched, resulting in dominant long wavelength trap states in the emission spectrum. Consequently, with the reduction of the sulfur precursor to $1: 3$, less quenching occurred causing the intensity of the band edge emission to increase, thereby exhibiting equal dominance with the surface defect state emissions. 


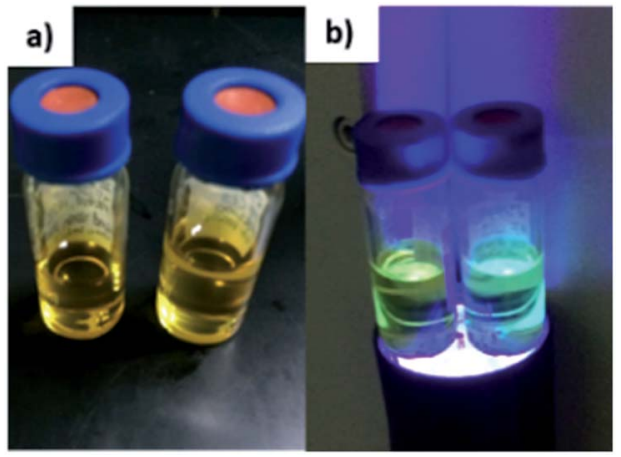

Fig. 8 Millifluidic chip synthesized CdS NCs seen under (a) ambient light and (b) upon illumination with ultraviolet (UV) light and emitting greenish-blue light due to band edge emissions.

The photoluminescence of the CdS NCs could be further enhanced by coating the NCs with zinc sulfide (ZnS) shells. ZnS is a wide band gap material which confines the exciton to the core of the NCs, thus improving the quantum yield of the NCs. Techniques such as successive ionic layer adsorption and reaction (SILAR) and thermal cycling with a single precursor (TC-SP) have been demonstrated to improve the quantum yield of the core-shell $\mathrm{CdS} / \mathrm{ZnS} \mathrm{NCs}$ from around $6 \%$ to $30 \%$ $\left(\right.$ SILAR) ${ }^{41,42}$ and $50 \%$ (TC-SP). ${ }^{41}$

Although the synthesis of CdS in miniature reaction vessels have been studied by many groups as shown earlier in Table 1, some have reported that the particles exhibited poor crystal quality, while others mostly reported cubic zinc blende structures. Based on the XRD measurements depicted in Fig. 10, in terms of the flow rate, we observed that at $5000 \mu \mathrm{l} \mathrm{min}^{-1}$ where the residence time is only $15 \mathrm{~s}$, some signal can still be captured. The presence of crystal lattice fringes in the high resolution TEM (HRTEM) and diffraction rings in the selected area electron diffraction (SAED) pattern for the NCs synthesized at $5000 \mu \mathrm{lmin}^{-1}$ as presented in Fig. 11b confirmed the existence of crystalline nanoparticles with interplanar lattice spacings of $2.07 \AA$ matching the $d$ spacings of the $\left\langle\begin{array}{llll}1 & 1 & 0\end{array}\right\rangle$ wurtzite planes and ( $\left.\begin{array}{lll}2 & 2 & 0\end{array}\right)$ zinc blende planes, and $3.4 \AA$ tallying with the $\left\langle\begin{array}{lll}1 & 0 & 0\end{array}\right)$ wurtzite $(d=3.58 \AA)$ and ( $\left.\begin{array}{lll}1 & 1 & 1\end{array}\right)$ zinc blende $(d=3.35$ $\AA)$ planes. The SAED pattern also revealed lattice plane distances of $1.1 \AA$ and $1.75 \AA$, which pointed to the wurtzite $\langle 3$

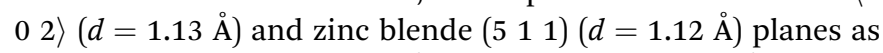
well as the $\left\langle\begin{array}{lll}1 & 1 & 2\end{array}\right\rangle(d=1.76 \AA)$ and $\left(\begin{array}{lll}3 & 1 & 1\end{array}\right)\left(\begin{array}{l}d=1.75 \AA \\ \text { A }\end{array}\right)$ planes. However, there are unique signatures such as the $2.07 \AA$ lattice spacing indicating the $\left\langle\begin{array}{lll}1 & 1 & 0\end{array}\right\rangle$ wurtzite structure and the diffraction ring for the $3.1 \AA\left\langle\begin{array}{lll}1 & 0 & 1\end{array}\right\rangle$ plane. This indicated the possibility of mixed phases being present in the CdS NCs with a dominant wurtzite crystal structure.

Subsequently as the flow rate increased, there were two main peaks at $44^{\circ}$ and $52^{\circ}$, both of which could be contributed by either the hexagonal wurtzite Greenockite $\left(43.7^{\circ}\right.$ and $51.9^{\circ}$ corresponding to $\left\langle\begin{array}{llll}1 & 1 & 0\end{array}\right\rangle$ and $\left\langle\begin{array}{llll}1 & 1 & 2\end{array}\right\rangle$ planes) or cubic zinc blende Hawleyite $\left(44.0^{\circ}\right.$ and $52.2^{\circ}$ corresponding to (2 $\left.2 \begin{array}{ll}2 & 0\end{array}\right)$ and $\left(\begin{array}{lll}3 & 1 & 1\end{array}\right)$ planes) structures. The subsequent emergence of a tiny peak at $36^{\circ}$ matching the $\left\langle\begin{array}{lll}1 & 0 & 2\end{array}\right\rangle$ plane of wurtzite evident in the XRD patterns for CdS NCs fabricated at flow rates of $750 \mu \mathrm{min}^{-1}$ and $3000 \mu \mathrm{lmin}^{-1}$ in Fig. 10a and $1: 12 \mathrm{Cd}: \mathrm{S}$ molar ratio in Fig. 10b provided compelling substantiation for the dominant wurtzite structure for these NCs.

\section{Bioimaging with macrophage cells}

QDs have been used for biological imaging as optical imaging probes, ${ }^{43}$ and near infrared imaging probes $^{\mathbf{4 4 , 4 5}}$ due to properties such as their wide absorption spectrum, narrow emission spectrum, and improved photostability compared to organic dyes. ${ }^{46}$ However, concerns involving the use of QDs include photoblinking and heavy metal toxicity. ${ }^{47}$ Here, we demonstrate fluorescence imaging of the RAW264.7 mice macrophage cell line with CdS NCs which have been ligand exchanged with mercaptosuccinic acid (MSA) for solubility in aqueous conditions. In this work, the CdS nanoparticles used for cellular a)

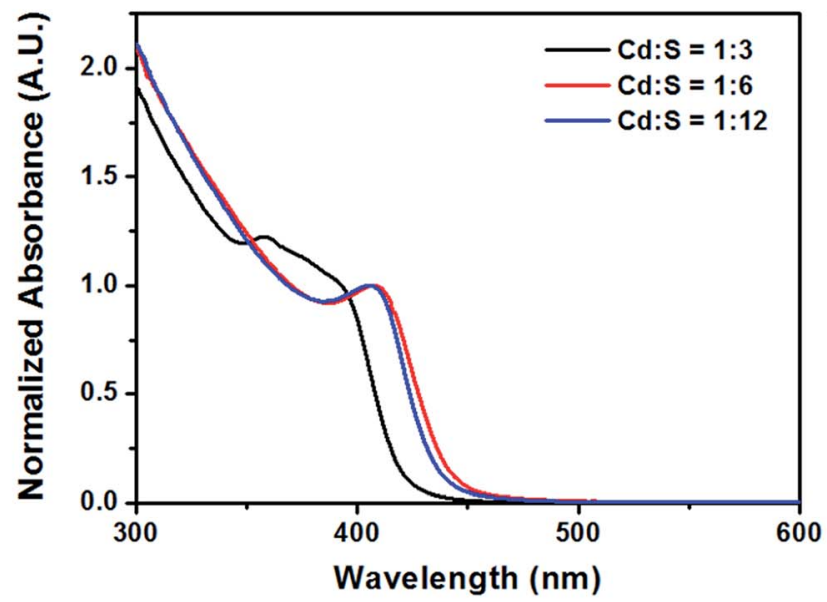

b)

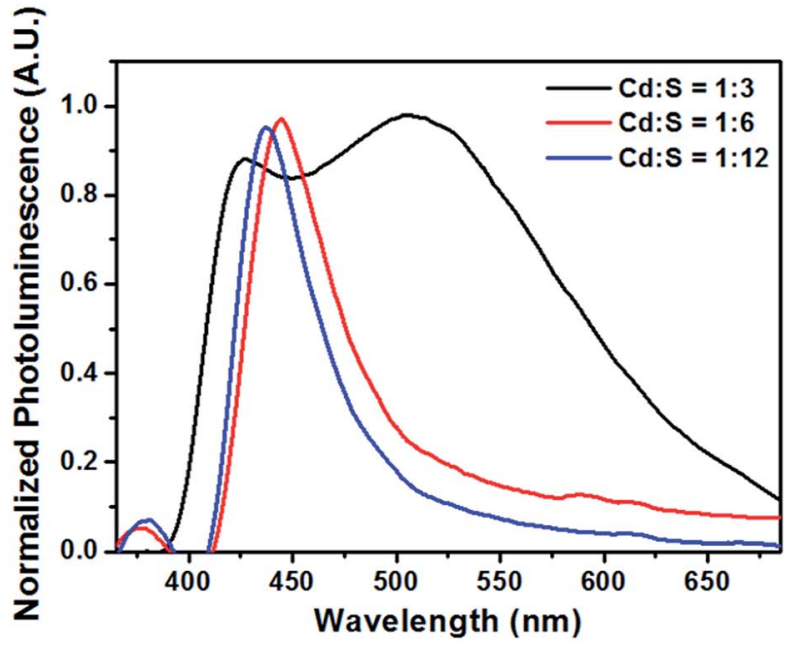

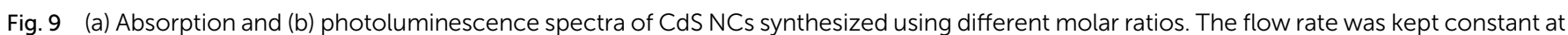
$1500 \mu \mathrm{lmin}^{-1}$. 
a)

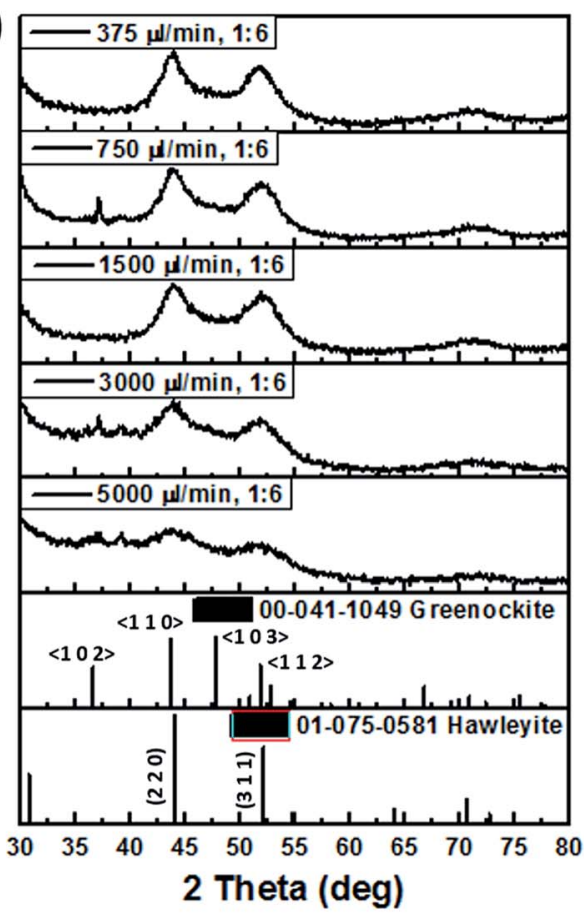

b)

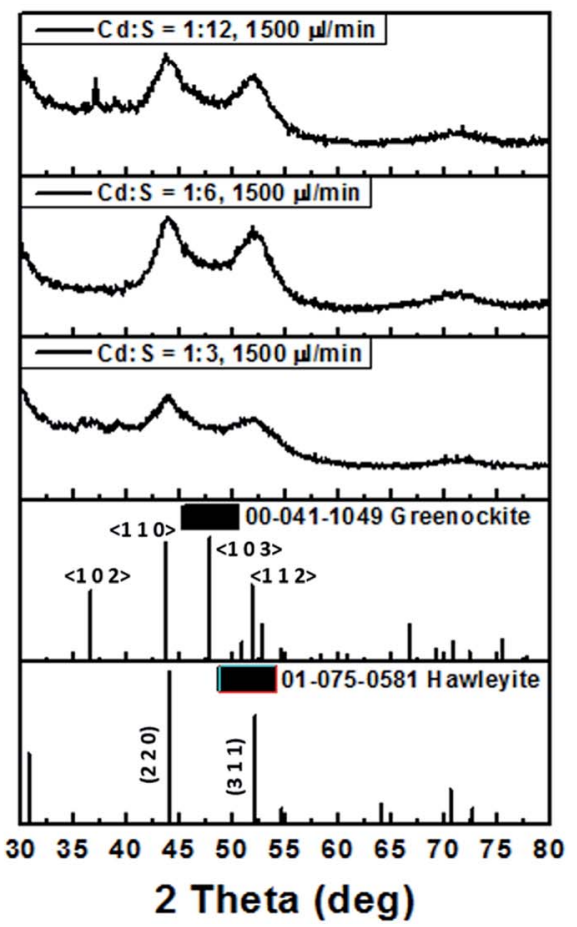

Fig. 10 Powered X-ray diffraction (XRD) patterns of millifluidic chip synthesized CdS at (a) different flow rates and (b) molar ratios.

imaging were not coated with any particular protein or biomolecules, only the mercaptosuccinic acid (MSA) ligand to make the nanoparticles biocompatible and facilitate cell uptake. For selective endocytosis, these nanoparticles can be further engineered by attaching antibodies and targeting moieties to the nanoparticle surface. These CdS-MSA NCs can be used as optical probes to monitor the activity of the nanoparticles and investigate the breakdown of CdS in the cells. We believe that the small dimensions of the millifluidic chip synthesized CdS NCs would serve as an excellent model to study the excretion pathway of the nanoparticles.

$3.27 \mu \mathrm{M}$ CdS-MSA NCs were added to RAW264.7 mice macrophage cells and incubated for 24 hours before imaging under ultraviolet (UV) light. The NCs were readily taken up by the macrophages as indicated by the numerous red regions observed from the dark field images depicted in Fig. 12. The
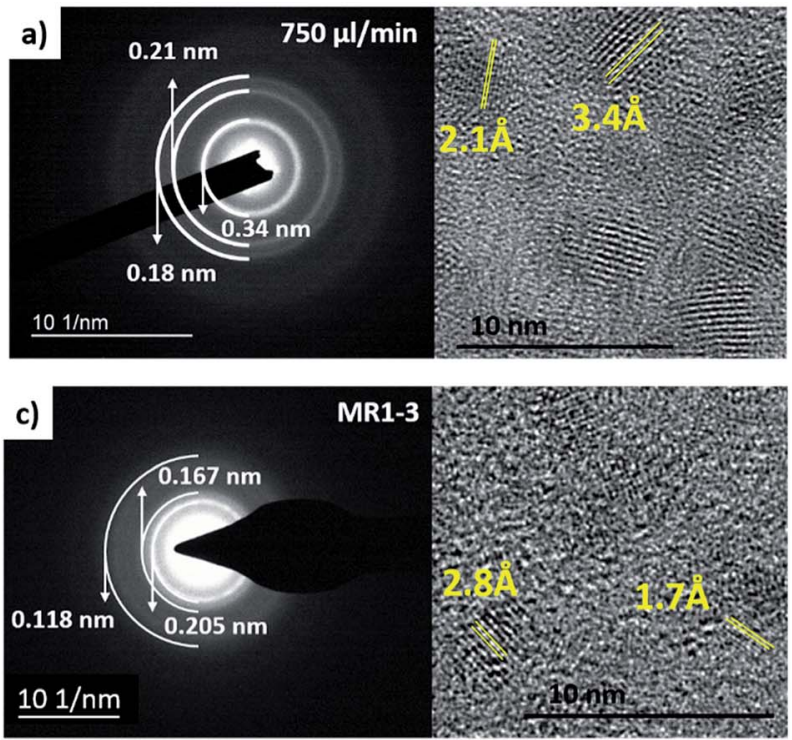

b)
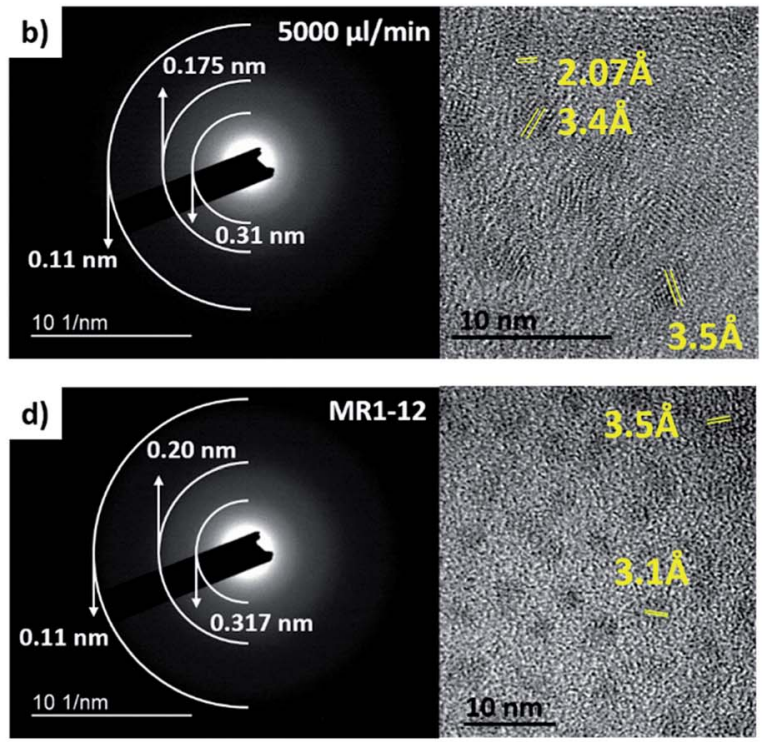

Fig. 11 Selected area electron diffraction (SAED) patterns and high resolution TEM (HRTEM) images of millifluidic chip synthesized CdS at different flow rates of (a) $750 \mu \mathrm{lmin}^{-1}$ and (b) $5000 \mu \mathrm{lmin}^{-1}$, and different Cd: S molar ratios of (c) $1: 3$ and (d) $1: 12$. 
Untreated Cells

Bright Field Image

\section{Dark Field \\ Fluorescence Image}

\subsection{7 $\mu \mathrm{mol} / \mathrm{I}$ CdS-MSA}
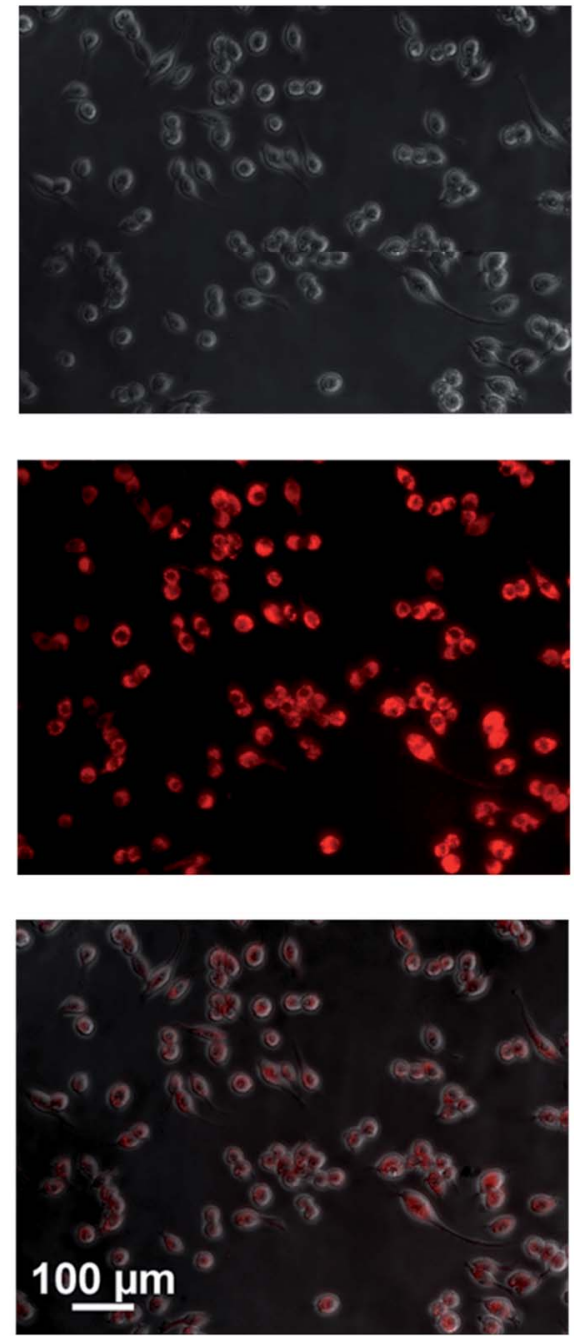

Fig. 12 In vitro bioimaging of RAW264.7 macrophage cells with and without CdS-MSA NCs. The red fluorescence observed is due to the emission of the CdS-MSA NCs upon illumination with ultraviolet (UV) light.

photoluminescence spectrum of the CdS-MSA NCs in Fig. 13 showed a peak at around $620 \mathrm{~nm}$. Therefore, the red fluorescence is indicative of the presence of CdS-MSA NCs ingested by the macrophages, while the control experiment shown in Fig. 12 exhibited no fluorescence for cells which were not treated (i.e. untreated) with the NCs.

Nanoparticles have large surface area to volume ratios, thus quantum confinement effects such as size, shape, surface charge, and surface passivation contribute significantly to the toxicity of the nanoparticles. Therefore, we believe that our millifluidic chip serves as an excellent platform to synthesize highly reproducible nanoparticles with good monodispersity, which would then serve as models for detailed studies on the effects of NC size, morphologies and surface modifications on nanoparticle toxicity.

A few strategies can be employed to reduce the toxicity of the NCs, one of which being coating the bare NCs with a zinc sulfide (ZnS) layer ${ }^{41,42}$ or encapsulation of these $\mathrm{NCs}^{48}$ to reduce leakage

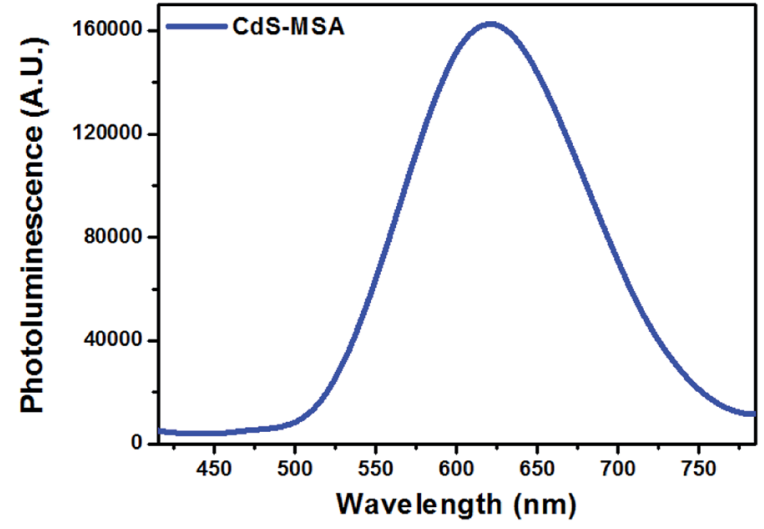

Fig. 13 Photoluminescence spectrum of ligand exchanged CdS-MSA nanocrystals. 
of free $\mathrm{Cd}^{2+}$ ions from the NC surface into the cellular environment. Free $\mathrm{Cd}^{2+}$ ions are highly toxic as they can generate reactive oxygen species (ROS), resulting in oxidative stress and cell apoptosis. Therefore, containment of the Cd core is crucial for biological applications of such cadmium based quantum dots.

\section{Conclusion}

In this work, we have demonstrated that CdS nanoparticles can be fabricated with ease and high reproducibility using a simple millifluidic chip. Changing the residence time (or injection flow rate) of the reactants in the millifluidic chip enabled precise control over nucleation and growth conditions and facilitated the synthesis of spherical CdS nanocrystals from as small as 2.4 $\pm 0.4 \mathrm{~nm}$ to $5.8 \pm 0.9 \mathrm{~nm}$ to multipod structures with arms spanning $6.7 \pm 2 \mathrm{~nm}$ long and $2.6 \pm 0.6 \mathrm{~nm}$ wide. The novelty in this work is the synthesis of highly reproducible, luminescent CdS nanocrystals with the presence of magic sized clusters. In addition, multipod structures were also observed in short reaction durations of less than 2 minutes. Lastly, we also showed that these luminescent nanocrystals can be employed as fluorescent probes for cell imaging studies. Therefore, we believe that there is great potential for millifluidic chip devices in the area of nanoparticle synthesis as they allow design flexibility to promote efficient mixing and provide stable environments suitable for nucleation and growth of nanoparticles.

\section{Acknowledgements}

This work was supported by the Singapore Ministry of Education (Grants Tier 2 MOE2010-T2-2-010 (M4020020.040 ARC2/11) and Tier 1 M4010360.040 RG29/10), NTU-NHG Innovation Collaboration Grant (No. M4061202.040), A*STAR Science and Engineering Research Council (No. M4070176.040), NEWRI seed funding (Grant No. NEWRI SF20140901) and School of Electrical and Electronic Engineering at NTU, The Hong Kong Polytechnic University (Project Grant: 1-ZE3A), the Research Grants Council of the Hong Kong Special Administrative Region, China (PolyU 25200914) and National Natural Science Foundation of China (61405169). The authors would like to thank Mr Jayaram s/o Raju for his assistance with this project.

\section{References}

1 J. Z. Zhang, J. Phys. Chem. B, 2000, 104, 7239-7253.

2 R. W. Meulenberg, J. R. I. Lee, A. Wolcott, J. Z. Zhang, L. J. Terminello and T. van Buuren, ACS Nano, 2009, 3, 325-330.

3 R. Luque and R. S. Varma, Sustainable Preparation of Metal Nanoparticles: Methods and Applications, The Royal Society of Chemistry, 2012.

4 K. Kalyanasundaram, E. Borgarello, D. Duonghong and M. Grätzel, Angew. Chem., Int. Ed. Engl., 1981, 20, 987-988.

5 K.-T. Yong, Y. Sahoo, M. T. Swihart and P. N. Prasad, J. Phys. Chem. C, 2007, 111, 2447-2458.

6 Z. A. Peng and X. Peng, J. Am. Chem. Soc., 2001, 123, 183-184.
7 X. Wang, Z. Feng, D. Fan, F. Fan and C. Li, Cryst. Growth Des., 2010, 10, 5312-5318.

8 S. Kar, S. K. Panda, B. Satpati, P. V. Satyam and S. Chaudhuri, J. Nanosci. Nanotechnol., 2006, 6, 771-776.

9 Y. Zou, D. Li and D. Yang, Nanoscale Res. Lett., 2011, 6, 1-6. 10 Y. C. Cao and J. Wang, J. Am. Chem. Soc., 2004, 126, 1433614337.

11 Y. Wada, H. Kuramoto, J. Anand, T. Kitamura, T. Sakata, H. Mori and S. Yanagida, J. Mater. Chem., 2001, 11, 19361940.

12 H. Yang, C. Huang, X. Li, R. Shi and K. Zhang, Mater. Chem. Phys., 2005, 90, 155-158.

13 V. Smyntyna, B. Semenenko, V. Skobeeva and N. Malushin, Beilstein J. Nanotechnol., 2014, 5, 355-359.

14 A. Giberti, B. Fabbri, A. Gaiardo, V. Guidi and C. Malagù, Appl. Phys. Lett., 2014, 104, 222102.

15 C. A. Rodríguez-Castañeda, P. M. Moreno-Romero, C. Martínez-Alonso and H. Hu, J. Nanomater., 2015, 2015, 10.

16 R. Sharma, S. Bhalerao and D. Gupta, Org. Electron., 2016, 33, 274-280.

17 N. Ma, J. Yang, K. M. Stewart and S. O. Kelley, Langmuir, 2007, 23, 12783-12787.

18 A. Gaiardo, B. Fabbri, V. Guidi, P. Bellutti, A. Giberti, S. Gherardi, L. Vanzetti, C. Malagù and G. Zonta, Sensors, 2016, 16, 296.

19 J. Joo, H. B. Na, T. Yu, J. H. Yu, Y. W. Kim, F. Wu, J. Z. Zhang and T. Hyeon, J. Am. Chem. Soc., 2003, 125, 11100-11105.

20 Y. Wu, C. Wadia, W. Ma, B. Sadtler and A. P. Alivisatos, Nano Lett., 2008, 8, 2551-2555.

21 I. Shestopalov, J. D. Tice and R. F. Ismagilov, Lab Chip, 2004, 4, 316-321.

22 L.-H. Hung, K. M. Choi, W.-Y. Tseng, Y.-C. Tan, K. J. Shea and A. P. Lee, Lab Chip, 2006, 6, 174-178.

23 H. Yang, W. Luan, Z. Wan, S.-t. Tu, W.-K. Yuan and Z. M. Wang, Cryst. Growth Des., 2009, 9, 4807-4813.

24 Z. Wan, H. Yang, W. Luan, S.-t. Tu and X. Zhou, Nanoscale Res. Lett., 2009, 5, 130-137.

25 M. S. Naughton, V. Kumar, Y. Bonita, K. Deshpande and P. J. A. Kenis, Nanoscale, 2015, 7, 15895-15903.

26 G. Chen, B. Yi, G. Zeng, Q. Niu, M. Yan, A. Chen, J. Du, J. Huang and Q. Zhang, Colloids Surf., B, 2014, 117, 199-205.

27 X. Zhu, D. Kumari, M. Huang and V. Achal, Mater. Des., 2016, 98, 209-214.

28 A. J. de Mello, Nature, 2006, 442, 394-402.

29 V. Hessel, H. Löwe and F. Schönfeld, Chem. Eng. Sci., 2005, 60, 2479-2501.

30 S. Pennathur, C. D. Meinhart and H. T. Soh, Lab Chip, 2008, 8, 20-22.

31 K. Sai Krishna, C. V. Navin, S. Biswas, V. Singh, K. Ham, G. L. Bovenkamp, C. S. Theegala, J. T. Miller, J. J. Spivey and C. S. S. R. Kumar, J. Am. Chem. Soc., 2013, 135, 54505456.

32 J.-I. Yoshida, H. Kim and A. Nagaki, ChemSusChem, 2011, 4, 331-340.

33 S. E. Lohse, J. R. Eller, S. T. Sivapalan, M. R. Plews and C. J. Murphy, ACS Nano, 2013, 7, 4135-4150. 
34 V. Mengeaud, J. Josserand and H. H. Girault, Anal. Chem., 2002, 74, 4279-4286.

35 T.-L. Cheung, L. Hong, N. Rao, C. Yang, L. Wang, W. J. Lai, P. H. J. Chong, W.-C. Law and K.-T. Yong, Nanoscale, 2016, 8, 6609-6622.

36 J.-I. Yoshida, Basics of Flow Microreactor Synthesis, Springer, 2015.

37 W. W. Yu, L. Qu, W. Guo and X. Peng, Chem. Mater., 2003, 15, 2854-2860.

38 S. M. Harrell, J. R. McBride and S. J. Rosenthal, Chem. Mater., 2013, 25, 1199-1210.

39 H. H.-Y. Wei, C. M. Evans, B. D. Swartz, A. J. Neukirch, J. Young, O. V. Prezhdo and T. D. Krauss, Nano Lett., 2012, 12, 4465-4471.

40 A. Veamatahau, B. Jiang, T. Seifert, S. Makuta, K. Latham, M. Kanehara, T. Teranishi and Y. Tachibana, Phys. Chem. Chem. Phys., 2015, 17, 2850-2858.

41 D. Chen, F. Zhao, H. Qi, M. Rutherford and X. Peng, Chem. Mater., 2010, 22, 1437-1444.
42 J. S. Steckel, J. P. Zimmer, S. Coe-Sullivan, N. E. Stott, V. Bulović and M. G. Bawendi, Angew. Chem., Int. Ed., 2004, 43, 2154-2158.

$43 \mathrm{~J} . \mathrm{Li}$ and J.-J. Zhu, Analyst, 2013, 138, 2506-2515.

44 T. Zako, M. Yoshimoto, H. Hyodo, H. Kishimoto, M. Ito, K. Kaneko, K. Soga and M. Maeda, Biomater. Sci., 2015, 3, 59-64.

45 E. I. Altınoğlu and J. H. Adair, Wiley Interdiscip. Rev.: Nanomed. Nanobiotechnol., 2010, 2, 461-477.

46 U. Resch-Genger, M. Grabolle, S. Cavaliere-Jaricot, R. Nitschke and T. Nann, Nat. Methods, 2008, 5, 763-775.

47 L. Yildirimer, N. T. K. Thanh, M. Loizidou and A. M. Seifalian, Nano Today, 2011, 6, 585-607.

48 S. Veeranarayanan, A. C. Poulose, M. S. Mohamed, Y. Nagaoka, S. Iwai, Y. Nakagame, S. Kashiwada, Y. Yoshida, T. Maekawa and D. S. Kumar, Int. J. Nanomed., 2012, 7, 3769-3786. 